\title{
Application of Yokohama System for Reporting Breast Fine Needle Aspiration Cytology in Correlation with Histopathological and Radiological Findings.
}

\author{
Murari. Apuroopa*, V. Kalyan Chakravarthy and D. Ranga rao \\ Dept of Pathology, Dr. Pinnamaneni Siddhartha Institute of Medical Sciences and Research Foundation, \\ Chinna Avutapalli, Gannavaram, AP, India
}

\begin{abstract}
Background: Breast cancer is the leading cause of cancer deaths among women worldwide. Fine needle aspiration cytology (FNAC) and breast ultrasound are pivotal in characterization of a breast lump as benign and malignant lesion. The main objectives were to categorise the Breast FNAC samples according to new system of reporting and to assess the Risk of malignancy (ROM), sensitivity, specificity, positive predictive value (PPV), negative predictive value (NPV) and diagnostic accuracy for all categories.

Methods: A Total of 900 FNAB cytology specimens from January 2017 to December 2018 were obtained. These were reclassified according to newly proposed IAC Yokohama system of reporting. The ROM, sensitivity, specificity, Positive Predictive Value (PPV), Negative Predictive Value (NPV) and diagnostic yield of Breast FNAC and also of ultrasound were calculated.

Result: Among 900 cases, $4.3 \%$ fall in category 1, 58\%- category 2, 17.7\%-category 3, 7.2\%- category 4 and $12.8 \%$ - category 5 , and correlated with corresponding radiological impression. Sensitivity, specificity, PPV, NPV and diagnostic accuracy of ultrasound were respectively $91.6 \%, 96.8 \%, 95.6 \%, 92.8 \%, 91.5 \%$ and those of FNAC respectively $94.59 \%, 98.9 \%, 98.59 \%, 95.74 \%, 96.97 \%$. ROM is $5.0 \%$ for category $1,1.2 \%$ for category $2,12.5 \%$ for category $3,93.65 \%$ for category 4 and $100 \%$ for category 5 .

Conclusion: IAC Yokohama system of reporting breast cytopathology serves as a common language to pathologist and clinician. Breast ultrasound is a non-invasive imaging-based technique and both these diagnostic tools can complement each other for patient diagnosis and management.
\end{abstract}

Keywords: Ultrasound, Yokohama, PPV, NPV.

\section{Introduction}

Breast cancer is the most common malignancy in women worldwide. It is the most frequent cause of cancer death in women in less developed countries and the second cause of cancer death in more developed regions ${ }^{[1]}$. Currently, the use of breast fine needle aspiration cytology (FNAC) varies greatly between hospitals and cities and between developed and developing countries. It offers many advantages such as it is minimally invasive, causes minimal physical and psychological discomfort, is highly acceptable to patients.

FNAC is a highly specific and sensitive test that accurately diagnoses benign and malignant lesions, and it is cost effective for the pre-operative diagnosis of palpable and ultrasound detected impalpable breast lesions. It also provides cell blocks for immunohistochemistry to identify prognostic indicators and smeared cells for polymerase chain reaction (PCR) and other potential molecular testing ${ }^{[2]}$. The overall diagnostic accuracy of this test can be improved by a good aspiration technique ${ }^{[3]}$. Whenever there is discrepancy between clinical examination, breast FNAC and breast ultrasound, breast biopsy is recommended to arrive at a final diagnosis. There is a need to correlate the radiological and cytological findings of breast lesions with histopathological diagnosis to evaluate the diagnostic accuracy of ultrasound and breast FNAC. Study aimed to correlate radiological and cytological findings of breast lesions with histopathological diagnosis.

In 2016, the International Academy of Cytology (IAC) executive council put together a breast group, which consists of cytopathologists, surgical pathologists, radiologists, surgeons and oncologists working in breast care, with the aim of producing a comprehensive and standardised approach to breast FNAC reporting. The breast group has decided to use a five category system used widely internationally. Category 1 - insufficient material, category 2 - benign, category 3 - atypical, probably benign, category 4 - suspicious for malignancy, category 5 - malignant.

\section{Aims and objectives}

To review and re-categorise the FNAC cases according to Yokohama system and correlate with radiological findings. 
To calculate the risk of malignancy (ROM) of each category.

To calculate the sensitivity, specificity, positive predictive value(PPV), negative predicitive value (NPV) and diagnostic accuracy for all categories.

\section{Materials and Methods}

All breast FNAC cases from 01-01-2017 to 31-12-2018 were retrieved from archive of pathology department, PSIMS\&RF. Matching histopathology samples were also retrieved. All the cytology cases were retrospectively categorised by newly proposed IAC Yokohama reporting system. Breast reporting system: categorised into five categories, 1- insufficient, 2-benign, 3-atypical, 4-suspicious of malignancy, 5-malignant. Ultrasound examination of the breast masses was done by a radiologist in the department of radio diagnosis. The transducer was gently applied and both transverse and longitudinal scans were taken. The scans included evaluating 4 features (i) Shape-Round/Oval or irregular.(ii) Margins Circumscribed / non circumscribed. (iii) Width- AP ratio $>1.4$ or $=1.4$ (iv) Echogenicity - Hyperechoic/Isoechoic or Hypoechoic. On the basis of these four features, diagnosis was made. The studies were reviewed and reported according to Breast Imaging-Reporting and Data System (BIRADS) protocol. The radiologist's report was recorded as radiological diagnosis. This study is approved by Institutional Ethical Committee, and got clearance certificate.

Statistical analysis is executed using Microsoft Excel 2011. ROM is defined for each category as the number of confirmed malignant cases / total number of cases in the diagnostic category. sensitivity, specificity, positive predictive value(PPV), negative predicitive value (NPV) and diagnostic accuracy are calculated for all categories

\section{Result}

A total of 900 cases were reviewed and categorised according to IAC Yokohama reporting system. Among 900 cases, 39 cases (4.3\%) fall under category 1, 522 cases $(58 \%)$ under category 2,160 cases $(17.7 \%)$ under category 3,63 cases $(7.2 \%)$ under category 4 and 116 cases $(12.8 \%)$ under category 5 (Fig 1), and we correlated these categories with corresponding radiological impression( Table 1). On breast ultrasonography 712 cases were diagnosed as benign breast disease (BIRADS I, II and III). 188 cases were diagnosed as suspicious for carcinoma or carcinoma of the breast (BIRADS IV, V). 2 cases were misdiagnosed false positively as carcinoma. Of these one was a cellular fibroadenoma and other was a radial scar with proliferative breast disease on histopathology.

Out of 900 cases histopathologic samples were available in 609 cases (Table 2). We compared the result of breast FNAC and breast ultrasound with matched histological diagnosis (Table 3). In the present study the sensitivity, specificity, positive predictive value, negative predictive value and diagnostic accuracy of ultrasound were respectively $91.6 \%, 96.8 \%, 95.6 \%, 92.8 \%$ and $91.5 \%$ (Table 4). ROM is calculated for each category of Yokohama system. ROM for each category was $5.0 \%$ for category $1,1.2 \%$ for category $2,12.5 \%$ for category $3,93.65 \%$ for category 4 and $100 \%$ for category 5 (Table 5). Sensitivity, specificity, positive predictive value, negative predictive value and diagnostic accuracy of FNAC were respectively $94.59 \%$, $98.9 \%, 98.59 \%, 95.74 \%$ and $96.97 \%$ (Table 6).

Table 1: correlation of Yokohama category with radiological findings.

\begin{tabular}{|l|l|l|l|}
\hline IAC Yokohama category & Number of cases FNAC & Radiological diagnosis & \\
\hline \multicolumn{2}{|l|}{} & BIRADS I, II, III & BIRADS IV, V \\
\hline 1 - Insufficient & 39 & 38 & 1 \\
\hline 2 - Benign & 522 & 516 & 6 \\
\hline 3 - Atypical probably benign & 160 & 153 & 7 \\
\hline 4 - Suspicious of malignancy & 63 & 3 & 60 \\
\hline 5 - Malignant & 116 & 2 & 114 \\
\hline
\end{tabular}

Table 2: correlation of Yokohama category with matched histopathology diagnosis.

\begin{tabular}{|l|l|l|l|l|}
\hline \multicolumn{2}{|l|}{ IAC Yokohama category } & Total number of cases (FNAC) & Matched histopathology cases \\
\hline \multicolumn{1}{|l|}{} & Number of cases & Benign & Malignant \\
\hline 1 - Insufficient & 39 & 20 & 19 & 1 \\
\hline 2 - Benign & 522 & 330 & 326 & 4 \\
\hline 3 - Atypical probably benign & 160 & 80 & 70 & 10 \\
\hline 4 - Suspicious of malignancy & 63 & 63 & 4 & 59 \\
\hline 5 - Malignant & 116 & 116 & -- & 116 \\
\hline Total & $\mathbf{9 0 0}$ & $\mathbf{6 0 9}$ & $\mathbf{4 1 9}$ & $\mathbf{1 9 0}$ \\
\hline
\end{tabular}


Table 3: Correlation of Yokohama category with matched histopathology diagnosis and radiodiagnosis.

\begin{tabular}{|l|l|l|l|l|l|}
\hline Yokohama Category & $\begin{array}{l}\text { No. of cases } \\
\text { FNAC }\end{array}$ & \multicolumn{2}{l|}{ Radio diagnosis No. of cases } & \multicolumn{2}{l|}{$\begin{array}{l}\text { Histopathology diagnosis No. of } \\
\text { cases }\end{array}$} \\
\hline & & BIRADS I,II,III & BIRADS IV,V & Benign & Malignant \\
\hline 1 - Insufficient & 20 & 19 & 1 & 19 & 1 \\
\hline 2 - Benign & 330 & 324 & 6 & 326 & 4 \\
\hline 3 - Atypical probably benign & 80 & 73 & 7 & 70 & 10 \\
\hline 4 - Suspicious of malignancy & 63 & 3 & 60 & 4 & 59 \\
\hline 5 - Malignant & 116 & 2 & 114 & --- & 116 \\
\hline
\end{tabular}

Table 4: Sensitivity, specificity, PPV, NPV and diagnostic accuracy rate of ultrasound.

\begin{tabular}{|l|l|}
\hline Statistical parameters & Percentage (\%) \\
\hline Sensitivity & 91.6 \\
\hline Specificity & 96.8 \\
\hline Positive predictive value & 95.6 \\
\hline Negative predictive value & 92.8 \\
\hline Diagnostic accuracy & 91.5 \\
\hline
\end{tabular}

Table 5: Risk of malignancy of different diagnostic categories.

\begin{tabular}{|l|l|}
\hline IAC Yokohama category & ROM \\
\hline Category 1 - Insufficient & $5.0 \%$ \\
\hline Category 2 - Benign & $1.2 \%$ \\
\hline Category 3 - Atypical, probably benign & $12.5 \%$ \\
\hline Category 4 - Suspicious for malignancy & $93.65 \%$ \\
\hline Category 5 - Malignant & $100 \%$ \\
\hline
\end{tabular}

Table 6: Sensitivity, specificity, PPV, NPV and diagnostic accuracy rate of breast cytology.

\begin{tabular}{|l|l|}
\hline Statistical parameters & Percentage (\%) \\
\hline Sensitivity & 94.59 \\
\hline Specificity & 98.9 \\
\hline Positive predictive value & 98.59 \\
\hline Negative predictive value & 95.74 \\
\hline Diagnostic accuracy & 96.97 \\
\hline
\end{tabular}

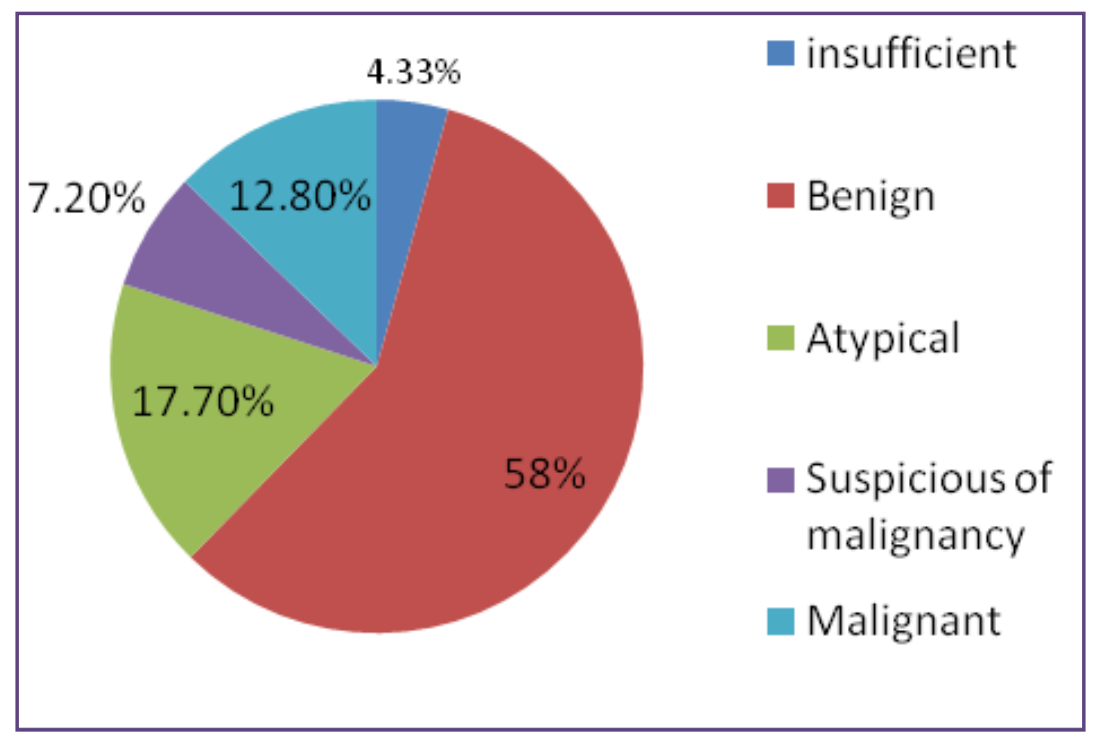

Fig. 1: Distribution of our samples according to IAC Yokohama system for reporting breast cytology. 


\section{Discussion}

Breast lesions encompass malignancies and many different benign lesions. Most women presenting with breast changes will be found to have a benign diagnosis ${ }^{[4]}$. In many countries triple assessment approach is advocated while assessing a breast mass, which combines clinical, radiologic and pathologic information to ensure accurate diagnosis and patient management ${ }^{[5,6]}$. Despite its many benefits like simplicity of the procedure, cost effectiveness, few complications, short turn around time several factors such as relatively high inadequate rate and suboptimal accuracy in some centres have led to criticism of FNAC ${ }^{[7,8,9]}$.

The use of a standardised reporting system will facilitate reproducibility of reports across institutions and the communication between pathologists and attending physicians with better patient care. This system will enhance clinicians use of breast FNAC and when appropriate core needle biopsy $(\mathrm{CNB})^{[10]}$.

In our study, we retrospectively re-categorised 900 breast FNAC slides in accordance with the proposed
IAC Yokohama reporting system and the distribution of our samples according to IAC Yokohama system are comparable to studies by Montezuma D et al., ${ }^{[11]}$ and Wong $S$ et al. ${ }^{[12]}$ (Table 7). we calculated the associated ROM for each category, ROM for category 1 is $5.0 \%$, adequate value for this category. Category 2 showed a low ROM 1.2\%, out of four false negative cases one corresponded to ductal carcinoma insitu, other three probably caused by error in sampling site of mass. Category 3 has ROM of $12.5 \%$, this category encompasses mainly benign lesions, but some cases will ultimately correspond to malignancy. ROM for category 4 is $93.6 \%$ and for category 5 it is $100 \%$ and our results are comparable with those obtained by Montezuma D etal ${ }^{[11]}$ (Table 8$)$.

In the present study the sensitivity, specificity, positive predictive value, negative predictive value and diagnostic accuracy of FNAC were respectively $95.9 \%, 97.89 \%$, $96.79 \%, 97.64 \%$ and $98.57 \%$. The results are comparable with Montezuma D et al., ${ }^{[11]}$ and Moschetta $\mathrm{M}$ et al., ${ }^{[14]}$ (Table:9)

Table: 7 The distribution of our samples according to IAC Yokohama system are comparable to studies by Montezuma D et al., and Wong $S$ et al.,

\begin{tabular}{|l|l|l|l|l|}
\hline Categories & $\begin{array}{l}\text { Montezuma D } \\
\text { et al., }\end{array}$ & $\begin{array}{l}\text { Wong S } \\
\text { et al., }\end{array}$ & $\begin{array}{l}\text { P112] } \\
\text { et al., }{ }^{[13]}\end{array}$ & Our study \\
\hline Insufficient & $209(5.77 \%)$ & $301(11 \%)$ & $22(5 \%)$ & $39(4.3 \%)$ \\
\hline Benign & $2660(73.38 \%)$ & $1937(72 \%)$ & $332(71 \%)$ & $522(58 \%)$ \\
\hline Atypical probably benign & $498(13.74 \%)$ & $117(4.3 \%)$ & $7(1 \%)$ & $160(17.7)$ \\
\hline Suspicious & $57(1.57 \%)$ & $59(2.2 \%)$ & $8(2 \%)$ & $63(7.2 \%)$ \\
\hline Malignant & $201(5.54 \%)$ & $278(10 \%)$ & $101(21 \%)$ & $116(12.8 \%)$ \\
\hline Total & $\mathbf{3 6 2 5}$ & $\mathbf{2 6 9 6}$ & $\mathbf{4 7 0}$ & $\mathbf{9 0 0}$ \\
\hline
\end{tabular}

Table 8: comparision of calculated ROM with other studies.

\begin{tabular}{|l|l|l|l|}
\hline IAC Yokohama category & Montezuma D et al[11] & Poornima V Kamatar et al., ${ }^{[13]}$ & Present study \\
\hline Category 1 - Insufficient & $4.8 \%$ & $0 \%$ & $5.0 \%$ \\
\hline Category 2 - Benign & $1.4 \%$ & $4 \%$ & $1.2 \%$ \\
\hline Category 3 - Atypical, probably benign & $13 \%$ & $66 \%$ & $12.5 \%$ \\
\hline Category 4 - Suspicious for malignancy & $97.1 \%$ & $83 \%$ & $93.65 \%$ \\
\hline Category 5 - Malignant & $100 \%$ & $99 \%$ & $100 \%$ \\
\hline
\end{tabular}

Table: 9 Comparision of sensitivity, specificity, positive predictive value, negative predictive value and diagnostic accuracy of FNAC with other studies and our study.

\begin{tabular}{|l|l|l|l|l|}
\hline Statistical parameters & Montezuma D et al & Moschetta M et al & Poornima V Kamatar et al., ${ }^{[13]}$ & Present study \\
\hline Sensitivity & $97.56 \%$ & $97 \%$ & $94.59 \%$ & $95.9 \%$, \\
\hline Specificity & $100 \%$ & $94 \%$ & $98.9 \%$ & $97.89 \%$, \\
\hline Positive predictive value & $100 \%$ & $91 \%$ & $98.59 \%$ & $96.79 \%$, \\
\hline Negative predictive value & $98.62 \%$ & $98 \%$ & $95.74 \%$ & $97.64 \%$ \\
\hline Diagnostic accuracy & $99.11 \%$ & $95 \%$ & $96.97 \%$ & $98.57 \%$ \\
\hline
\end{tabular}


Ultrasound is useful in evaluation of breast lesions, it can be helpful to physicians in predicting the likelihood of cancer. Breast ultrasound is now a well established method and several studies have suggested that sonomammography can be useful in differentiating between benign and malignant lesions presenting as breast masses ${ }^{[15]}$. The purpose of this study was to compare the result of breast FNAC and breast ultrasound with histological diagnosis to assess its diagnostic accuracy. Early screening and diagnosis of breast lesions and categorization into different groups of breast pathology can be helpful in accurate management of the breast lesions.

In the present study the sensitivity, specificity, positive predictive value, negative predictive value and diagnostic accuracy of sonomammography were respectively $91.6 \%$, $96.8 \%, 95.6 \%, 92.8 \%$ and $91.5 \%$. In a study done by Wasan et al ${ }^{[16]}$ breast ultrasound had a sensitivity of $100 \%$, specificity of $91.6 \%$ which is comparable to our study. Benign lesions of the breast were more readily diagnosed by ultrasound than malignant lesions. According to Nandan

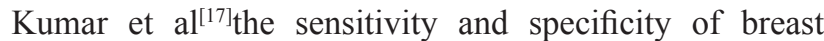
ultrasound in categorising breast lesions as benign and malignant was $85.4 \%$ and $89.31 \%$, which is slightly lesser compared to our study. Our study suggest the use of FNAC standardised report in conjunction with imaging findings which yields very high positive and negative predictive values, and provides a solid basis for management decisions in breast lesions. According to our knowledge not many studies are there in the literature to compare with our study.

\section{Conclusion}

In line with prior categorization schemes, such as the Bethesda System for Reporting Thyroid Cytopathology, the newly proposed IAC Yokohama System for Reporting Breast Cytopathology represents a simple system that allows greater diagnostic clarity and, consequently, better communication between pathologists and attending clinicians. Breast ultrasound is a non-invasive imaging based technique and both these diagnostic tools can complement each other for patient diagnosis and management. Furthermore, recent advances in both these techniques like Immunocytochemistry, imaging guided FNAC and Doppler in sonomammography may increase their accuracy.

\section{Abbreviations}

FNAC - Fine Needle Aspiraton Cytology

\section{PCR - Polymerase chain reaction}

IAC - International Academy of Cytology

ROM - Risk of malignancy
PPV - Positive predictive value

NPV - Negative predictive value

BI-RADS - Breast Imaging-Reporting and Data System

CNB - Core needle biopsy

Funding

None

Competing Interests

None Declared

Reference

1. Ferlay J, Soerjomataram I, Ervik M, Dikshit R, Eser S, Mathers C, et al. GLOBOCAN 2012: Estimated Cancer Incidence, Mortality and Prevalence Worldwide in 2012 v1.0: IARC CancerBase No. 11. Lyon: International Agency for Research on Cancer; 2013.

2. Schmitt F, Vielh P. Fine needle aspiration cytology samples: a good source of material for evaluating biomarkers in breast cancer. Histopathology. 2015;66:314-3150.

3. McManus, DT. \& Anderson, N.H. Fine needle aspiration cytology of the breast. Current DiagnosticPathology 2001;7:262-271Anderson BO. Fine needle aspiration for breast cancer diagnosis: one size does not fit all. J Natl Compr Canc Netw. 2016;14;599-600.

4. Rungruang B, Kelley JL 3rd. Benign breast diseases: epidemiology, evaluation, and management. Clin Obstet Gynecol. 2011 Mar; 54(1): 110-24.

5. Kreuzer G, Boquoi E. Aspiration biopsy cytology, mammography and clinical exploration: a modern set up in diagnosis of tumors of the breast. Acta Cytol. 1976 Jul-Aug; 20(4): 319-23.

6. Wai CJ, Al-Mubarak G, Homer MJ, Goldkamp A, Samenfeld-Specht M, Lee Y, et al. A modified triple test for palpable breast masses: the value of ultrasound and core needle biopsy. Ann Surg Oncol. 2013 Mar; 20(3): 850- 5.

7. Wells CA, Perera R, White FE, Domizio P. Fine needle aspiration cytology in the UK breast screening programme: a national audit of results. Breast. 1999 Oct; 8(5): 261-6.

8. Pisano ED, Fajardo LL, Tsimikas J, Sneige N, Frable WJ, Gatsonis CA, et al. Rate of insufficient samples for fine-needle aspiration for nonpalpable breast lesions in a multicenter clinical trial: The Radiologic Diagnostic Oncology Group 5 Study. The RDOG5 investigators. Cancer. 1998 Feb; 82(4): 679-88.

9. Pisano ED, Fajardo LL, Caudry DJ, Sneige N, Frable WJ, Berg WA, et al. Fine-needle aspiration biopsy of nonpalpable breast lesions in a multicenter clinical trial: results from the radiologic diagnostic oncology group V. Radiology. 2001 Jun; 219(3): 785-92.

10. Field AS, Schmitt F, Vielh P. IAC standardized reporting of breast fine-needle aspiration biopsy cytology. Acta Cytol. 2017; 61(1): 3-6. 
11. Montezuma D, Malheiros D, Schmitt FC. Breast Fine needle aspiration biopsy [7] cytology using the newly proposed IAC Yokohama system for reporting breast cytopathology: The experience of a single institution. Acta Cytol. 2019;63(Suppl.4):274-79.

12. Wong S, Rickard M, Earls P, Arnold L, Bako B, Field AS. The International [8] Academy of Cytology Yokohama System for reporting breast fine needle aspiration biopsy cytopathology: A single institutional retrospective study of the application of the system categories and the impact of rapid onsite evaluation. Acta Cytol. 2019;63(Suppl.4):280-91.

13. Kamatar PV et al., Breast Fine needle Aspiration Biopsy Cytology Reporting using International Academy of Cytology Yokohama SystemTwo Year Retrospective Study in Tertiary Care Centre in Southern India. National Journal of Laboratory Medicine. 2019 Oct, Vol-8(3): PO01-PO03.
14. Moschetta M. Comparison between fine needle aspiration cytology (FNAC) [9] and core needle biopsy (CNB) in the diagnosis of breast lesions. G Chir- J Surg [Internet]. 2014 [cited 2019 Jul 17]; Available from: http://www.giornalechirurgia. it/common/php/portiere. php?ID=73366646a0b094bb6a480507c74c39ea

15. Heinig J, Witteler R, Schmitz R, Kiesel L, Steinhard J. Accuracy of classification of breast ultrasound findings based on criteria used for BI-RADS. Ultrasound Obstet Gynecol. 2008; 32:573-78.

16. Wasan et al: Non Palpable Breast Mass. Mustansiriya Medical Journal Volume 15 Issue 1 April 201.

17. Nandan Kumar1, Shilpa $\mathrm{N}$ et al. Mammographic And Sonomammographic Evaluation Of Breast Masses With Pathological Correlation. Innovative Journal of Medical and Health Science 2016;6:132 - 135.

*Corresponding author:

Dr. Murari. Apuroopa, No: 13- 129, FF-1, Kasturi towers, Paala bazaar, near prathibha high school, Prasadampadu, Vijayawada - 521108

Email: m urariroopa42@gmail.com

Financial or other Competing Interests: None. 